\begin{abstract}
¿Cómo citar este artículo?
Lastre Meza, K., Aguas de la Puente, L. y Parra Sarmiento, K. (enero-abril, 2020). Efectos del programa para la estimulación y el mantenimiento cognitivo en demencias (Córtex) en una institución de Sincelejo (Colombia). Revista Virtual Universidad Católica del Norte, (59), 133-149. doi: https://doi.org/10.35575/rvucn.n59a8
\end{abstract}

\title{
| Efectos del programa para la estimulación y el mantenimiento cognitivo en demencias (Córtex) en una institución de Sincelejo (Colombia)
}

Effects of the implementation of the program for the stimulation and cognitive maintenance in dementia (Cortex) in an institution of Sincelejo (Colombia)

\section{Karina Lastre Meza}

Magister en Neuropsicología y Educación Universidad de Sucre

Colombia

karina.lastre@unisucre.edu.co

Orcid: http://orcid.org/0000-0002-0713-1578

\author{
Lina Aguas de la Puente \\ Fonoaudióloga \\ Universidad de Sucre \\ Colombia \\ linaaguas26@gmail.com \\ Orcid: https://orcid.org/0000-0002-2807-7160
}

\section{Katty Parra Sarmiento}

Fonoaudióloga

Universidad de Sucre

Colombia

kathyparra1@outlook.es

Orcid: https://orcid.org/0000-0001-9241-9383

Recibido: 05 de julio de 2019 Evaluado: 18 de septiembre de 2019

Tipo de artículo: Investigación científica y tecnológica.

Aprobado: 10 de diciembre de 2019

\section{Resumen}

Se determinaron los efectos de la implementación del programa de estimulación y mantenimiento cognitivo en la demencia (CORTEX) en una población con deterioro cognitivo, a través de un estudio cuantitativo, explicativo, diseño cuasi-experimental, con prebruepa- postprueba en un grupo de estudio, en una muestra de diez (10) adultos mayores, seleccionados según criterios de inclusión y exclusión. En un primer momento, se aplicó la prueba Minimental y Moca como evaluación inicial; seguidamente, se ejecutó el programa de estimulación CORTEX durante cuatro meses, concluyendo con un postest con los mismos instrumentos. Los resultados demostraron que existen diferencias estadísticamente significativas en las funciones cognitivas de los pacientes cuando reciben entrenamiento cognitivo.

Palabras clave: Atención, Deterioro cognitivo, Lenguaje, Memoria. 


\section{| Abstract}

The effects of the implementation of the Program of Cognitive Stimulation and Maintenance in Dementia (CORTEX) in a population with cognitive impairment were determined, through a quantitative, explanatory study, quasi-experimental design with pretest- posttest in ten (10) older adults according to inclusion and exclusion criteria. Initially, it was applied the Minimental and Moca tests as initial evaluation, then the CORTEX stimulation program was run for four months. The results demonstrated that there are statistically significant differences in the cognitive functions of patients when they receive cognitive training.

Keywords: Kttention, Cognitive impairment, Language, Memory.

\section{| Introducción}

El ser humano pasa por diferentes etapas o ciclos de vida, que inician con la concepción y terminan con la muerte. Este proceso de desarrollo avanza paulatinamente con la niñez, luego se progresa de manera gradual y continúa a la adolescencia, periodo crucial con evolución biológica y psicológica que da paso a la adultez; en esta etapa, las experiencias y aprendizajes proporcionan estabilidad y seguridad, terminando más o menos a los 60 o 65 años, dando inicio a la etapa del adulto mayor o vejez.

Conceptualmente, se ha entendido la vejez como edad avanzada, edad tardía o tercera edad; constituye, según Medellin y Tascon (1995) el periodo de vida comprendido entre los 60 y 65 años de edad hasta la muerte, a partir del cual concluye el desarrollo de todas las esferas del individuo y trae consigo una serie de cambios biológicos, psicológicos y sociales, caracterizados por el declive de todas las facultades humanas.

Actualmente, el deterioro cognitivo leve (DCL) afecta a un gran número de adultos mayores y es considerado uno de los motivos de consulta más frecuente en neurología, al punto de considerarse un problema de salud pública. Ferri et al. (2005) definen el deterioro cognitivo leve $(\mathrm{DCL})$ como la pérdida o alteración de las funciones mentales, tales como memoria, orientación, lenguaje, reconocimiento visual, capacidad de juicio y otras, sin afectar actividades de la vida diaria; además, es considerada una etapa transicional entre el envejecimiento normal y las primeras fases de demencia; esta última definida por American Psychiatric Association (1995) como:

El desarrollo de múltiples déficits cognoscitivos que incluyen un deterioro de la memoria y, al menos, una de las siguientes alteraciones cognoscitivas: afasia, apraxia, agnosia o una alteración de la capacidad de ejecución. La alteración es lo suficientemente grave como para interferir de forma significativa las actividades laborales y sociales y puede representar un déficit respecto al mayor nivel previo de actividad del sujeto. (p. 140). 
En el ámbito mundial, de acuerdo a la Organización Mundial de la Salud -OMS- (2017) se calcula que hay 47 millones de personas con demencia y deterioro en el mundo, y se prevé que esta cifra aumente a 75 millones de aquí a 2030. Se estima que el número de casos se triplicará para 2050; "La prevalencia de vida de la demencia en personas de 60 años y más, está entre el $5 \%$ y el $8 \%$, se presenta tempranamente en el $9 \%$ de los casos (en menores de 65 años: demencia precoz)" (Ministerio de Salud y Protección Social, 2017, p. 4).

Por su parte, en Colombia el estudio neuroepidemiológico nacional, en el 2003, reveló un índice de prevalencia de casos de demencia en un 13,1 de los habitantes (Pradilla, Vesga, Boris y León-Sarmiento, 2003); otros estudios más específicos en personas mayores de 50 años, arrojaron cifras entre 1,3 y 5,4\% (Díaz-Cabezas, Ruano-Restrepo, Chacón-Cardona y Vera-González, 2006), y en adultos mayores de 60 años una prevalencia global de demencia de 23.6 (Goodling, Amaya, Parra y Ríos, 2006). En los últimos años, la Encuesta Nacional de Salud, Bienestar y Envejecimiento, SABE (Ministerio de Salud y Protección Social -MinSalud-, 2015) reportó una prevalencia de demencia del 9,4\% (IC95\%: 7.7-11.4), que incrementó con la edad (mayores de 85 años, prevalencia de 57,4\%). Al igual que los datos suministrados por el Sistema Integral de Información de la Protección Social -SISPRO- (2017), la prevalencia es mayor en mujeres (10.7\%) que en hombres (8.1\%). Otro dato interesante, suministrado por la Encuesta, fue la alta prevalencia en la región del Atlántico y los departamentos que la conforman (proporción de 10.1 hombres; 15.5 mujeres); se halló mayormente en estrato socioeconómico 1 y en personas sin ningún nivel educativo (MinSalud, 2015, p.6).

Especificamente hablando del departamento de Sucre, no existe una estadística que revele la prevalencia de casos con deterioro cognitivo; sin embargo, el Plan de Desarrollo Departamental (2016-2019) deja claro el grado de representatividad poblacional que tienen los adultos mayores en el departamento; al respecto refiere:

\begin{abstract}
Que la población adulta mayor representa el 8.6\% del total de los sucreños. El número de adultos mayores en el departamento de Sucre acrecentó 1184 con relación 2012 al 2011, y sigue una tendencia a aumentar en 1315 en el 2013, con relación al 2012, mientras que, en 2014, con relación al 2013, tuvo un aumento leve de 1173; la relación hombre mujer corresponde a la misma distribución de la población total y se destaca que las principales dificultades para el desarrollo de actividades cotidianas son de tipo visual, para desplazarse, pensar y memorizar. (p. 88).
\end{abstract}

De acuerdo a lo anterior, se puede inferir un crecimiento paulatino de la población adulta mayor sucreña, que puede manifestar un declive en el rendimiento global cognoscitivo y, por tanto, presentar deterioro cognitivo como una de las patologías más frecuentes en este grupo de edad, cuyos efectos repercuten en su calidad de vida; no obstante, son pocas las iniciativas investigativas que se han publicado en el departamento de Sucre, sobre el abordaje del deterioro cognitivo en los adultos mayores. Sumado a esto, el plan de desarrollo en mención, también deja entrever la carencia en el departamento de centros geriátricos especializados que ofrezcan atención integral a la población con deterioro cognitivo; las opciones que existen son limitadas a los asilos, que aumentan la desidia familiar, la carencia afectiva, y la actividad 
mental y física. Por tanto, se infiere que la oferta de servicios y bienes para esta población es restringida y con muchas barreras de acceso para la satisfacción de sus necesidades básicas.

En este marco lógico, resulta pertinente el desarrollo de la presente investigación que busca determinar los efectos del programa de estimulación y mantenimiento cognitivo en demencias (CORTEX) en una población con deterioro cognitivo en Sincelejo, lo cual implica, primeramente, identificar las características cognitivas de los pacientes, a partir de una valoración inicial; seguido, ejecutar el programa de estimulación en la población; y como último, comparar las características cognitivas antes y después de la intervención del programa.

El aporte de la investigación estará dado en validar la ejecución del programa CORTEX en una población colombiana, al estimular, activar e intervenir funciones cognitivas superiores afectadas, como el lenguaje, las gnosias, praxias, atención y memoria, en aras de ralentizar su declive; igualmente, se pretende tributar a la teoría del deterioro cognitivo en la vejez y que el estudio se constituya en un antecedente para futuras investigaciones.

Después de las consideraciones anteriores, la implementación de programas de estimulación en poblaciones con deterioro cognitivo ha sido objeto de investigación científica en el contexto internacional y nacional; sin embargo, no existe suficiente evidencia científica que demuestre el uso del programa de estimulación y mantenimiento cognitivo CORTEX; solo se cita el estudio de Jiménez (2015), quien intentó comprobar la eficacia de la estimulación cognitiva en un paciente con alteración cognitivo-emocional; para ello se apoyó en varios programas, entre los que incluyó el CORTEX de Maroto, Blanco, Berrocosa, Sánchez y Saavedra (2012), cuyos resultados confirmaron el hecho de que la estimulación cognitiva es una clave importante en la ralentización del deterioro cognitivo y, por ende, en la elevación del estado de ánimo de los ancianos.

De manera cronológica, también se da crédito a otros autores que estudiaron el deterioro cognitivo e intentaron intervenirlo; tal es el caso de Garamendi, Delgado y Amaya (2010), Bae et al. (2019), García-Sevilla, Fernández, Fuentes, López y Moreno (2014), Quintana Hernández y Miró Barrachina (2015), y Lastre Meza (2015), quienes ejecutaron investigaciones orientadas principalmente a programas de entrenamiento en personas mayores, sobre diversas funciones cognitivas, entre ellas la memoria, el lenguaje, funciones visoespaciales, razonamiento, lectura y escritura, además de potenciar la coherencia funcional y la integración personal, así como medir la efectividad de intervenciones multidimensionales que combinan actividades fisicas, cognitivas y sociales, desarrolladas para promover la actividades básicas, instrumentales o avanzadas y, por ende, la función cognitiva en adultos mayores con deterioro cognitivo.

Otros autores (por ejemplo, D'Antonio et al., 2019; Fabbri et al., 2018; O'Caoimh et al., 2019; Zhang et al., 2019) realizaron revisiones sistemáticas de los tratamientos disponibles en el manejo del deterioro cognitivo; la búsqueda arrojó que intervenciones como electroterapia, ejercicios físicos, acupresión-acupuntura, atención plena, terapia cognitiva conductual y técnicas más novedosas, como prácticas mindfulness, telerehabilitacion, neurofeeback, entrenamiento cognitivo en el hogar, versus entrenamiento computarizado, se emplean para 
prevenir o retrasar el avance del deterioro cognitivo en sujetos.

Recientemente, autores como Piras et al. (2017) expresan que la terapia de estimulación cognitiva, como intervención psicosocial, potencia aspectos como el funcionamiento cognitivo, calidad de vida, estado de ánimo, comportamiento, actividades de la vida diaria en pacientes con demencia. Su estudio reveló la tendencia de la memoria de trabajo a mejorar luego de dicha estimulación; caso similar ocurre con tareas de cálculo con dígitos y la percepción de la calidad de vida. Aunado a ello, Félix, Ribeiro \& Maia (2019) agregan que la estimulación cognitiva suele ser más efectiva en modalidades personalizadas, con un enfoque de atención centrado en la persona, y con una relación más estrecha entre terapeuta- paciente, a partir de la cual se promueva un mayor compromiso, participación, aceptación y estabilización del estado de ánimo del paciente.

\section{| Método}

Esta investigación se enmarcó dentro del enfoque cuantitativo, con un tipo de estudio explicativo (Hernández Sampieri, Fernández Collado y Baptista Lucio, 2014). Además, se asumió un diseño cuasi-experimental con prebruepa- posprueba en un solo grupo estudio.

Dentro de esta investigación se asumieron como variables de estudio: el programa de estimulación cognitiva CORTEX (variable independiente) y las funciones cognitivas de memoria, lenguaje, atención, Praxias (variable dependiente). Se asumieron dos hipótesis de estudio:

Ho: No existen diferencias en las funciones cognitivas antes y después del entrenamiento con el programa CORTEX.

H1: Si existen diferencias en las funciones cognitivas antes y después del entrenamiento con el programa CORTEX.

\section{Participantes}

La población universo estuvo compuesta por treinta (30) adultos mayores de un Asilo en la ciudad de Sincelejo, en edades comprendidas entre los 60 y los 85 años; para la selección de la muestra se aplicó un muestreo intencional por criterios. I) De inclusión: adultos mayores pertenecientes a la comunidad del Asilo; ii) De exclusión: adultos con déficit cognitivo, con pérdida visual y auditiva, sin prótesis correctiva. De esta manera, la muestra quedó reducida a 20 participantes del estudio.

A esta muestra se les aplicó la evaluación pretest con los instrumentos screnning Minimental y Moca, y siguiendo los requerimientos que establece el programa CORTEX, el cual se divide en dos grandes niveles; el primero o Nivel 1 reúne las fichas y ejercicios destinados a personas con 
deterioro cognitivo moderado, y toma como referencia la puntuación del Examen cognoscitivo Minimental, con aproximadamente entre 16 y 23 puntos. El Nivel 2 reúne las fichas y estrategias destinadas a personas con una puntuación aproximada entre 10 y 19 puntos en el examen Minimental.

Así, el grupo de estudio lo conformaron 10 adultos mayores que integraron los niveles 1 y 2 de intervención del Programa CORTEX, distribuidos de la siguiente manera:

Nivel I: 8 pacientes (alcanzaron puntuaciones entre 16 y 23)

Nivel 2: 2 pacientes (alcanzaron puntuaciones entre 10 y 19)

\section{Herramientas}

Como instrumentos de recolección de la información se utilizaron la prueba Screnning Minimental (Lobo, Escobar, Ezquerra y Seva Díaz, 1980), el cual analizó 12 aspectos: orientación temporal, orientación espacial, atención, calculo, fijación, memoria, nominación, repetición, comprensión, lectura, escritura y dibujo. Como puntos de corte o valoración se tiene: función cognoscitiva Normal (27-30 puntos), Sospecha Patológica (26-23 puntos), Deterioro (12-23 puntos), Demencia (9-12 puntos), fase terminal ( $<9$ puntos).

Así mismo, se aplicó la Evaluación Cognitiva Montreal Moca (Nasreddine, Phillips y Bédirian, 2016) concebida para evaluar las disfunciones cognitivas leves y examinar las siguientes habilidades: atención, concentración, funciones ejecutivas (incluyendo la capacidad de abstracción), memoria, lenguaje, capacidades visuoconstructivas, cálculo y orientación. Cabe anotar que este instrumento se utilizó como una escala meramente orientativa para comparar los resultados.

\section{Procedimiento}

La investigación se desarrolló a través de una serie de fases:

Acercamiento a la comunidad: con los directivos del Asilo de la ciudad de Sincelejo, se dio a conocer toda la información concerniente a la investigación y cada una de sus etapas; posterior a esto se diligenció el consentimiento informado para participar en el estudio, basándose en las indicaciones dadas por el Ministerio de Salud, en su Resolución 8430 (1993), por la cual se establecen las normas científicas, técnicas y administrativas para la investigación en salud. Así mismo, se cumplió con las disposiciones legales vigentes para investigación en comunidades y las normas del comité de bioética.

Trabajo de campo: en esta etapa se procedió a la aplicación de los tamizajes o instrumentos de recolección de la información: la prueba Minimental y Moca. Estas se aplicaron de manera individual, con un tiempo aproximado de 40 a 60 minutos, y se eligió de acuerdo a los puntajes 
alcanzados el grupo de estudio.

Ejecución del programa: posterior a la aplicación de la evaluación inicial se desarrolló el programa de estimulación y mantenimiento cognitivo CORTEX, de los autores Maroto et al. (2012), el cual se desarrolló teniendo en cuenta la metodología planteada por los autores; tuvo una duración de cuatro (4) meses, para un total de 30 sesiones, que fueron ejecutadas 3 veces a la semana, con una intensidad horaria de 1 hora; cabe anotar que el programa establece que la sesión dure 3 horas, pero se modificó por requerimiento institucional. Se realizaron varias actividades, no similares entre sí, que incidían en diferentes funciones; así mismo, las planificaciones se alternaron, tal como indica el protocolo, con otras actividades desarrolladas en la institución para mantener el interés en los usuarios. Ahora bien, en la ejecución de la sesión se llevaron en físico las fichas del programa, las cuales traen las instrucciones que se tienen que hacer en cada actividad, que son suficientemente claras para que el propio sujeto las lea y trabaje de manera autónoma; sin embargo, los investigadores guiaban al paciente mientras trabajaba, proponiendo tareas y proporcionándole la ayuda que podían necesitar. Por último, para la ejecución de cada sesión se emplearon estrategias de refuerzo, control de estímulos, modelado y aprendizaje vicario, moldeamiento, extinción y refuerzo diferencial, sugeridas por el programa CORTEX.

Análisis de la información: se analizaron los resultados generados, a través del empleo del programa informático SPSS Statistics, versión 23, coherente con los instrumentos de investigación. En un primer momento, se utilizó estadística descriptiva con el cálculo de máximo, mínimo, media y desviación estándar en el análisis de las puntuaciones brutas de cada batería empleada. Para las comparaciones intregrupo (entre la evaluación antes y después del programa) se empleó estadística no paramétrica, mediante la prueba de suma de rangos de Wilcoxon, debido a la distribución anormal de los datos, y de esta manera estimar si existen diferencias estadísticamente significativas. La prueba manejó un nivel de significancia del 5\%.

\section{| Resultados}

\section{Características cognitivas de la población}




\section{Tabla 1}

Resultados obtenidos en instrumentos Moca y Minimental

\begin{tabular}{cccccc}
\hline \multicolumn{5}{c}{ Estadísticos descriptivos Moca } \\
\hline Hab. Visoespacial & 20 &, 00 & 6,00 & $\mathbf{1 , 4 5 0 0}$ & 1,63755 \\
Función Ejecutiva & & & & & \\
Atención & 20 &, 00 & 6,00 & 2,0500 & 1,53811 \\
Lenguaje & 20 &, 00 & 3,00 & $\mathbf{1 , 2 0 0 0}$ & 1,00525 \\
Abstracción & 20 &, 00 & 2,00 & $\mathbf{1 , 1 0 0 0}$ &, 91191 \\
Identificación & 20 &, 00 & 3,00 & 1,8000 & 1,00525 \\
Recuerdo diferido & 20 &, 00 & 5,00 &, 9500 & 1,82021 \\
Orientación & 20 &, 00 & 6,00 & 3,7000 & 2,67739 \\
& Estadísticos descriptivos Minimental & \\
Orientación & $\mathrm{N}$ & Mínimo & Máximo & Media & Desviación estándar \\
Temporal & 20 &, 00 & 5,00 & 3,1000 & 1,91669 \\
Orientación Espacial & 20 &, 00 & 5,00 & 4,2500 & 1,48235 \\
Fijación & 20 & 2,00 & 3,00 & 2,9000 &, 30779 \\
Atención y Calculo & 20 &, 00 & 5,00 & $\mathbf{2 , 3 0 0 0}$ & 2,43007 \\
Memoria & 20 &, 00 & 3,00 & $\mathbf{1 , 0 0 0 0}$ & 1,12390 \\
Nominación & 20 &, 00 & 2,00 & 1,8500 &, 48936 \\
Repetición & 20 &, 00 & 2,00 & $\mathbf{8 5 0 0}$ &, 48936 \\
Comprensión & 20 &, 00 & 3,00 & 2,7500 &, 78640 \\
Lectura & 20 &, 00 & 3,00 & $\mathbf{5 5 0 0}$ &, 75915 \\
Escritura & 20 &, 00 & 1,00 & $\mathbf{3 0 0 0}$ &, 47016 \\
Dibujo & 0 &, 00 & 1,00 &, 0500 &, 22361 \\
\hline
\end{tabular}

Nota elaboración propia a partir de las salidas del software SPSS Statistics, versión 23.

Como se evidencia en la tabla 1, se registran los resultados obtenidos en una muestra ( $\mathrm{n}: 20)$ de participantes para las pruebas screnning Minimental y Moca, sus máximos y mínimos, medias y desviaciones estándar. Nótese, en cuanto a los resultados obtenidos, para prueba cribado de Moca, los participantes mostraron puntuaciones por debajo de la media esperada en tareas que implican poner en actividad habilidades visoespaciales y función ejecutiva, lenguaje, abstracción y recuerdo diferido. Para el caso de los resultados hallados en la prueba cribado Minimental, se evidenció puntuaciones por debajo de la media esperada en dominios 
como atención y cálculo mental, memoria y repetición. Por último, el rendimiento fue nulo para actividades de código lectoescrito, como leer y comprender frases sencillas y escribir una frase coherente.

A partir de estos resultados se analizó la puntuación global del Minimental, encontrando que 2 pacientes alcanzaron una puntuación normal (27-30 puntos), 7 pacientes se ubicaron en deterioro cognitivo leve (26-23puntos), 8 pacientes en deterioro moderado (12-23 puntos) y 3 en deterioro cognitivo severo (9-12 puntos).

Con estos resultados se procedió a la conformación de los grupos de intervención para el entrenamiento cognitivo (CORTEX); se tuvo en cuenta 2 niveles de intervención: el primero destinado a personas que califiquen entre 16 y 23 puntos en la Prueba Minimental, y el segundo nivel para personas con aproximadamente entre 10 y 19 puntos en el mismo instrumento.

De esta manera, la muestra a intervenir fueron 10 pacientes, de los cuales 8 clasificaron para el nivel I, y 2 para el nivel II de intervención; el grupo restante obtuvo puntuaciones superiores a 23 puntos, y por tanto no aplican según los criterios del programa CORTEX para recibir el entrenamiento cognitivo.

\section{| Ejecución del programa de entrenamiento cognitivo CORTEX}

La intervención del CORTEX se desarrolló a partir de los meses de julio, agosto, septiembre y octubre de 2018, con un total de 30 sesiones trabajadas; tres sesiones por semana con una intensidad de una hora. Esta se desarrolló en las instalaciones de un Asilo en la ciudad de Sincelejo-Sucre, en jornada vespertina, y se trabajaron las siguientes áreas:

Lenguaje: ejercicios que incidieron en la capacidad de denominación, comprensión, lenguaje automático, fluidez verbal, la repetición, la categorización, el texto libre, la comprensión lectora y, de forma indirecta, también en la memoria semántica.

Atención: ejercicios de atención focal, atención sostenida, atención dividida, amplitud visoespacial, atención inhibida.

Praxias: actividades como la realización de gestos o mímica por orden o imitación; además, praxias constructivas relacionadas con tareas de construcción de modelos o dibujos a la orden; también, se incluyeron ejercicios que combinaban la motricidad fina con elementos de coordinación visual.

Memoria: memoria inmediata, memoria a largo plazo, memoria de trabajo, memoria episódica, semántica y procedural o procedimental, donde se implementaron ejercicios de 


\section{EFECTOS DEL PROGRAMA}

recuerdo y de reconocimiento.

Al término de las sesiones de estimulación y mantenimiento cognitivo se aplicó nuevamente el Test Minimental y Moca, además se realizó una comparación de las evaluaciones iníciales y finales.

\section{| Resultados obtenidos antes y después del programa de Entrenamiento CORTEX}

Para la comprobación del tercer objetivo, el cual buscaba comparar los resultados antes y después de la implementación del programa CORTEX, se empleó estadística inferencial; se procedió inicialmente a comprobar la normalidad de la distribución de los datos arrojados por los instrumentos Moca y Minimental, mediante la aplicación de la prueba Shapiro-Wilk, por ser una muestra menor a 50 datos; con ello se prueba la siguiente hipótesis:

Ho: Los datos se distribuye normalmente

Ha: Los datos NO se distribuye normalmente

El resultado de la prueba Shapiro-Wilk, reportó un valor de 0.153, y como este valor es mayor que 0.05 se demuestra que no existe una distribución normal de los datos ( $p>0.05$ ). A partir de lo anterior, se escoge como estadístico la prueba de rangos con signo de Wilcoxon, prueba no paramétrica que se utiliza para saber si existen diferencias antes y después del entrenamiento cognitivo. 


\section{EFECTOS DEL PROGRAMA}

Tabla 2

Resultados de la prueba de rangos con signo de Wilcoxon (T)

\begin{tabular}{lll}
\hline & \multicolumn{2}{c}{ Moca } \\
\cline { 2 - 3 } & Antes & Después \\
\hline Media & 6,7000 & 17,0000 \\
Desviación Estándar & 4,11096 & 5,90668 \\
Varianza & 16,900 & 34,889 \\
Z(K-S) & $-2,807^{\mathrm{b}}$ & \\
P(Valor) &, $005 \quad$ Minimental & \\
& \multicolumn{1}{c}{ Antes } & Después \\
Media & 16,6000 & 22,1000 \\
Desviación Estándar & 3,40588 & 4,77144 \\
Varianza & 11,600 & 22,767 \\
Z(K-S) & $-2,814^{\mathrm{b}}$ & \\
P(Valor) &, 005 & \\
\hline Nota: elaboracion & & \\
\hline
\end{tabular}

Nota: elaboración propia a partir de las salidas del software SPSS Statistics, versión 23.

La tabla 2 muestra el análisis de los resultados globales por cada instrumento, obtenidos antes y después del entrenamiento cognitivo en ambos instrumentos, por lo cual se rechaza la hipótesis nula y se asume la hipótesis alterna, concluyendo además que existen diferencias en las funciones cognitivas de los pacientes antes y después de recibir el entrenamiento con el programa CORTEX, y dichas diferencias son estadísticamente significativas (T: (10): p: 0.05).

\section{| Discusión}

El objetivo general del estudio fue determinar los efectos de la implementación del programa para la estimulación y el mantenimiento cognitivo en demencias (CORTEX) en una población con deterioro cognitivo en un centro geriátrico.

En este orden de ideas, y como muestra el análisis descriptivo para el primer objetivo, sobre la identificación de las características cognitivas de la población objeto de estudio, la información deja ver el perfil neurológico característico de un proceso de deterioro cognitivo en la población estudiada. De la aplicación de las pruebas screnning Minimental y Moca se observó el compromiso o alteración de uno o más dominios cognitivos; los más deteriorados 
en este caso son la memoria episódica, explicita verbal y de trabajo, seguida de las funciones de ejecución visoespacial, orientación temporal, alteraciones en el lenguaje en tareas específicas de fluidez y denominación. Al comparar con otras investigaciones y la teoría, se encuentra similitud en los hallazgos; si bien Álvaro (2018) expresa que el deterioro en la vejez puede afectar varias esferas cognitivas de manera progresiva, dentro de estas suele mencionar no solo a la memoria, sino además el lenguaje, las habilidades visoespaciales, la manipulativas o práxicas, de reconocimiento, las de abstracción, las numéricas, de organización y planificación. Sumado, De la Serna (2003) sostiene que las funciones constructivas que incluyen planear, ejecutar y evaluar secuencias complejas de conducta se ven afectadas por el deterioro cognitivo en la vejez, al igual que las tareas que requieren rapidez; también, los errores son más notables y las tareas visoespaciales (topográficas), como leer mapas, reconocer y reproducir figuras complejas o poco conocidas, presentan mayor dificultad.

Los resultados del presente estudio confirmaron en la población de adultos mayores estudiada, que el dominio cognitivo más afectado es la memoria episódica o de trabajo, aspecto que repercute en otras funciones cognitivas como el lenguaje en esfera proposicional; al mismo tiempo, el paciente pierde conexión espaciotemporal con la realidad y el entorno, lo que da lugar a fracasos en actividades de su cotidianidad. Los hallazgos son coincidentes con referentes teóricos como los De la Serna (2003), quien plantea que, con el deterioro cognitivo, producto de la vejez, se cursa una disminución de la capacidad inhibitoria o problemas con la memoria a corto plazo; también, las habilidades pragmáticas se ven afectadas con la reducción de oportunidades para hablar; estos problemas pueden muchas veces proceder de fallos en la visión y la audición

En cuanto al segundo objetivo, sobre la ejecución del programa de entrenamiento cognitivo CORTEX, se puede afirmar que luego de seleccionada la población se logró estimular, activar y mantener las funciones cognitivas de memoria, lenguaje, atención y praxias. Además, se alcanzaron resultados favorables comprobables estadísticamente con un antes y después; si se analiza, la meta lograda no tiene un carácter curativo ni milagroso, más bien, como señalan Pino y Escárcega (2016), consiste en mantener las funciones mentales superiores con procedimientos terapéuticos que estimulen capacidades cognoscitivas, emocionales, sociales y fisicas, de manera integral, para lentificar el deterioro cognoscitivo y funcional y, por tanto, mitigar la desestabilización del enfermo con su entorno. El abordaje deberá centrarse en el paciente y tener en cuenta el ambiente, la familia y el cuidador.

La mayoría de las intervenciones cognitivas se inspiran en estrategias didácticas fáciles de emplear, no solo por el profesional de cabecera, sino por el familiar y cuidador; al respecto, las actividades trabajadas con el programa de entrenamiento CORTEX se fundamentaron en técnicas de facilitación, como la repetición, ayudas mnemotécnicas, ejercicios prácticos y ecológicos, de fluidez verbal y denominación, los cuales buscan rescatar en el paciente habilidades como pensar, recordar, organizar información; además, dichas estrategias prácticas también han sido utilizadas en otras investigaciones con resultados positivos (García-Sevilla et al., 2014; Muñoz González, 2018; Pino y Escárcega, 2016), que dan cuenta de su utilidad y beneficio en la capacidad de orientación espacial, recuerdo inmediato y lenguaje.

Así mismo, en la ejecución del programa CORTEX también se empleó como estrategia de 
apoyo la repetición reiterada; Álvaro (2018), Madueño y De Sebastián (2018) afirman que la repetición es una de las habilidades más preservadas en el paciente con deterioro; por tanto, puede servir como estrategia compensatoria, pues las capacidades de aprendizaje se mantienen, aunque sean más lentas, debido a los fallos de memoria inmediata y de trabajo; pero si la información se adquiere de forma más lenta y mediante repetición se consolida igual que en personas más jóvenes. Lo anterior, ayuda a la representación mnemónica de conceptos, vocablos, estructuras sintácticas, lo que disminuye de manera considerable la anomia en el paciente con demencia, junto con las parafasias fonológicas y semánticas.

La investigación también comprobó la relación estadísticamente significativa ( $P$ : 0.05) entre el entrenamiento con el programa CORTEX y los rendimientos en la postprueba, con los tamices Minimental y Moca, lo cual confirma el hecho de que todos aquellos programas que estén dirigidos a la estimulación y mantenimiento de las funciones cognitivas pueden mitigar o paliar los efectos propios del deterioro cognitivo en individuos. Maroto et al. (2012) señalan que cada actividad dirigida a estimular o poner en marcha una determinada función primaria (atención, lenguaje, memoria, entre otras) puede incidir en funciones secundarias, o incluso terciarias, que indirectamente también se benefician de la estimulación (p. 64). Autores como Kinsella et al. (2016), Lam et al.(2015), Nakatsuka et al. (2015) y Rozo, Rodríguez, Montenegro y Dorado (2016), a través de sus investigaciones, demostraron que las intervenciones cognitivas, mediante grupos de memoria, sesiones colectivas y actividad fisica, dirigidas a grupos con envejecimiento normal, deterioro cognitivo y demencia, pueden ser beneficiosas para mantener la salud cognitiva y mejorar el rendimiento de la memoria; sin embargo, se observan beneficios más modestos para los adultos mayores con deterioro cognitivo.

Con el presente estudio, se puede afirmar que el objetivo de mantener y conservar durante el mayor tiempo posible las capacidades cognitivas de personas afectadas por algún tipo de demencia se cumplió en la población objeto de estudio, lo que valida una vez más el programa de entrenamiento de funciones cognitivas CORTEX. Tal como señala la OMS (2017),
(...) la terapia de estimulación cognitiva, como la participación en un rango de actividades con el fin de mejorar el funcionamiento social y cognitivo, es una estrategia crítica para prevenir y revertir el declive de la capacidad cognitiva, y consecuentemente, prevenir discapacidades funcionales y dependencia en la vejez. (p.14)

Así las cosas, toda intervención cognitiva que se dirija a poblaciones con algún tipo de deterioro cognitivo o demencia, coadyuvará a mantener la actividad cerebral, conservando la conexión con el entorno y su realidad.

De otro lado, al analizar los resultados y comprobar los efectos benéficos del programa de entrenamiento CORTEX para poblaciones con algún tipo de deterioro cognitivo, se afirma su validez y utilidad como herramienta terapéutica en la intervención de las funciones cognitivas en la vejez, y por tanto podrá tener un impacto significativo si se logra articular con las políticas de desarrollo social, programas y protocolos de atención integral en centros geriátricos, hogares de paso u otras instituciones que atiendan la población longeva. 
De igual forma, con los resultados obtenidos en este trabajo, y de acuerdo con los hallazgos revisados, se tributa a la teoría del envejecimiento cognitivo, al contemplar programas de intervención como estrategias y mecanismos compensatorios que posibiliten la prolongación de los niveles de funcionalidad cognitiva, competencia social y comunicativa en la vejez.

\section{| Conclusiones}

Del presente estudio se concluye que, en cuanto al primer objetivo sobre las características cognitivas, el estudio demostró que la población objeto de estudio, luego de ser evaluada, mostró un perfil característico de deterioro cognitivo con un declive en funciones cognitivas, como la memoria de trabajo, ejecución visoespacial, lenguaje, orientación temporal, atención y cálculo; el rendimiento fue nulo para actividades de código lectoescrito, hecho que confirma una vez más lo soportado en la teoría y otras investigaciones.

De la ejecución del programa de intervención CORTEX se puede concluir que se dieron cambios positivos en las áreas del lenguaje, memoria, atención y Praxias. Al término de las 30 sesiones la evaluación final arrojó cambios favorables en las funciones cognitivas de los pacientes cuando reciben el entrenamiento con el programa CORTEX, y dichas diferencias son estadisticamente significativas; por tanto, se resalta la validez y efectividad del entrenamiento cognitivo, que dependerá en gran medida de la frecuencia de las sesiones, la duración de la sesión y el tiempo general de intervención.

La investigación permitió establecer estadísticamente una relación significativa entre el entrenamiento con el programa CORTEX y los rendimientos en la postprueba con los tamices Minimental y Moca; sin embargo, cabe analizar, a partir de la metodología, algunas limitaciones de estudio, entre ellas la población evaluada e intervenida, que debido a los criterios de inclusión y exclusión se redujo significativamente; sin embargo, se recomienda para futuras investigaciones ampliar la población. También, se reconoce como limitación el tiempo de la intervención, el cual debería prolongarse longitudinalmente, con la finalidad de observar mejores resultados en todas las áreas intervenidas.

Finalmente, por intereses investigativos en el presente estudio solo se trabajaron 4 módulos del programa de entrenamiento CORTEX (Atención, Lenguaje, Gnosias, Praxias); resultaría interesante para probar su validez y confiabilidad, trabajar todo el programa e incluir los módulos de Cálculo, Funciones ejecutivas, Memoria, y Orientación. 


\section{| Referencias}

Álvaro, L. C. (2018). Competencia en demencia: manual de uso clinico. Colombia: Editorial Médica Panamericana.

American Psychiatric Association. (1995). DSM-IV-TR: Manual diagnóstico y estadístico de los trastornos mentales. Barcelona: MASSON

Bae, S., Lee, S., Lee, S., Jung, S., Makino, K., Harada, K., ... \& Shimada, H. (2019). The effect of a multicomponent intervention to promote community activity on cognitive function in older adults with mild cognitive impairment: A randomized controlled trial. Complementary Therapies in Medicine, 42, 164-169. Doi: /10.1016/j.ctim.2018.11.011.

D'Antonio, J., Simon-Pearson, L., Goldberg, T., Sneed, R. J., Rushia, S., Kerner, N.... \& Devanand, D. (2019). Cognitive training and neuroplasticity in cognitive impairment (COGIT): protocol for a two-site, blinded, randomised, controlled treatment trial. BMJ Open, 9(8), e028536. Doi:10.1136/ bmjopen-2018-028536.

De la Serna, I. (2003). La vejez desconocida. Una mirada desde la biología a la cultura. Madrid, España: Díaz Santos, S.A.

Díaz-Cabezas, R., Ruano-Restrepo, M. I., Chacón-Cardona, J. A. y Vera-Gonzalez, A. (2006). Perfil neuroepidemiológico en la zona centro del departamento de Caldas (Colombia), años 2004-2005. Revista de Neurología, 43(11), 646-52.

Fabbri, L., Mosca, I. E., Gerli, F., Martini, L., Pancani, S., Lucidi, G., ... GOAL Working Group. (2018). The Games for Older Adults Active Life (GOAL) Project for People With Mild Cognitive Impairment and Vascular Cognitive Impairment: A Study Protocol for a Randomized Controlled Trial. Frontiers in Neurology, 9, 1-9. https://doi.org/10.3389/fneur.2018.01040

Félix, S. B., Ribeiro, O. \& Maia, H. (2019). Personalized Cognitive Stimulation through Personhood: A Case Report on Dementia Diagnosis Acceptance and Therapeutic Engagement. Clinical Gerontologist, 1-7. Doi:10.1080/07317115.2019.1648349

Ferri, C. P., Prince, M., Brayne, C., Brodaty, H., Fratiglioni, L., Ganguli, M., ... Jorm, A. (2005). Global prevalence of dementia: a Delphi consensus study. The lancet, 366(9503), 2112-2117.

Garamendi, F., Delgado, D. A. y Amaya, M. A. (2010). Programa de entrenamiento cognitivo en adultos mayores. Revista Mexicana de Medicina Física y Rehabilitación, 22(1), 26-31.

García-Sevilla, J., Fernández, P. J., Fuentes, L. J., López, J. J. y Moreno, M. J. (2014). Estudio comparativo de dos programas de entrenamiento de la memoria en personas mayores con quejas subjetivas de memoria: un análisis preliminar. Anales de Psicología, 30(1), 337-345.

Gobernancion de Sucre. (2016). Plan Departamental de Desarrollo de Sucre, 2016-2019, Sucre progresa en paz. Recuperado de http://sucre.micolombiadigital.gov.co/sites/sucre/content/- 
files/000023/1140_plan-departamental-de-desarrollo-20162019.pdf

Goodling, M., Amaya, E., Parra, M. y Ríos, A. (2006). Prevalencia de las demencias en el municipio de Neiva 2003-2005. Acta Neurológica Colombiana, 22(3), 243-248.

Hernández Sampieri, R., Fernández Collado, C. y Baptista Lucio, P. (2014). Metodología de la investigación. México, D.F.: McGraw-Hill Education.

Jiménez, S. P. (2015). La influencia de la estimulación cognitiva en la alteración cognitivo-emocional: Un estudio de caso único. Revista Neurama, 2(2).

Kinsella, G. J., Ames, D., Storey, E., Ong, B., Pike, K. E., Saling, M. M.,... \& Rand, E. (2016). Strategies for improving memory: a randomized trial of memory groups for older people, including those with mild cognitive impairment. Journal of Alzheimer's Disease, 49(1), 31-43.

Lam, L. C., Chan, W. C., Leung, T., Fung, A. W. \& Leung, E. M. (2015). Would older adults with mild cognitive impairment adhere to and benefit from a structured lifestyle activity intervention to enhance cognition?: a cluster randomized controlled trial. PLoS One, 10(3), 1-17.

Lastre Meza, K. (2015). Implementación de un programa de estimulación del lenguaje en adultos mayores con envejecimiento comunicativo normal en la ciudad de Sincelejo, Sucre (Proyecto de investigación). Universidad de Sucre. Colombia.

Lobo, A., Escobar, V., Ezquerra, J. y Seva Díaz, A. (1980). "El Mini-Examen Cognoscitivo"(Un test sencillo, práctico, para detectar alteraciones intelectuales en pacientes psiquiátricos). Revista de psiquiatría y Psicología Médica, 14(5), 39-57.

Madueño, L. y De Sebastián, J. V. (2018). Manual práctico de patología del lenguaje: evaluación e intervención en adultos y niños. Barcelona, España: Editorial UOC.

Maroto, V., Blanco, J., Berrocosa, T., Sánchez. y Saavedra, C. (2012). CÓRTEX. Programa para la Estimulación y el Mantenimiento Cognitivo en Demencias. España: TEA Ediciones, S.A.

Medellin, G. y Tascon C. E. (1995). Atención primaria en salud. Crecimiento y desarrollo del ser humano. Tomo II: Edad Escolar a Adulto Mayor. Bogotá, Colombia: Editora Guadalupe Ltda

Ministerio de Salud y Proteccion Social -MINSALUD-.(1993). Resolucion 8430, por la cual se establecen las normas científicas, técnicas y administrativas para la investigación en salud. Recuperado de https://www.minsalud.gov.co/sites/rid/Lists/BibliotecaDigital/RIDE/DE/DIJ/RESOLUCION-8430-DE-1993.PDF

Ministerio de Salud y Proteccion Social -MINSALUD-. (2015). Encuesta Nacional de Salud, Bienestar y Envejecimiento, SABE. Bogotá, Colombia: MINSALUD.

Ministerio de Salud y Protección Social -MINSALUD-. (2017). Boletín de salud mental demencia. Recuperado de https://www.minsalud.gov.co/sites/rid/Lists/BibliotecaDigital/RIDE/VS/PP/ENT/Boletin-demencia-salud-mental.pdf

Muñoz González, D. A. (2018). La estimulación cognitiva como estrategia para la atención 


\section{EFECTOS DEL PROGRAMA}

\section{Efectos del programa | Revista virtual}

psicogerontológica a los adultos mayores con demencia. Revista Cubana de Salud Pública, 44(3), 1-8.

Nakatsuka, M., Nakamura, K., Hamanosono, R., Takahashi, Y., Kasai, M., Sato, Y., ... \& Meguro, K. (2015). A cluster randomized controlled trial of nonpharmacological interventions for old-old subjects with a Clinical Dementia Rating of 0.5: the Kurihara Project. Dementia and geriatric cognitive disorders extra, 5(2), 221-232.

Nasreddine, Z. S, Phillips, N. A. y Bédirian, V. (2016). Evaluacion cognitiva Montreal MOCA. Montreal cognitive assessment (E/C). España: Librería especializada Olejnik.

O'Caoimh, R., Mannion, H., Sezgin, D., O'Donovan, M. R., Liew, A. \& Molloy, D. W. (2019). Non-pharmacological treatments for sleep disturbance in mild cognitive impairment and dementia: A systematic review and meta-analysis. Maturitas, 127, 82-94. Doi:10.1016/j.maturitas.2019.06.007

Organización Mundial de la Salud -OMS-. (2017). 10 datos sobre la demencia. Recuperado de http://www.who.int/features/factfiles/dementia/es/.

Pino, M. B. y Escárcega, M. V. (2016). Abordaje no farmacológico de las demencias. Archivos de Neurociencias, 20(1) 95-102.

Piras, F., Carbone, E., Faggian, S., Salvalaio, E., Gardini, S. \& Borella, E. (2017). Efficacy of cognitive stimulation therapy for older adults with vascular dementia. Dementia \& Neuropsychologia, 11(4), 434-441. Doi:10.1590/1980-57642016dn11-040014

Pradilla, A. D., Vesga, A., Boris, E. y León-Sarmiento, F. E. (2003). Estudio neuroepidemiológico nacional (EPINEURO) colombiano. Revista Panamericana de Salud Pública, 14, 104-111

Quintana Hernández, D. J. y Miró Barrachina, M. T. (2015). Estimulación basada en mindfulness para personas mayores con enfermedad de alzheimer $u$ otras demencias. Papeles del Psicólogo, 36(3), 207-215.

Rozo, V., Rodríguez, O., Montenegro, Z. y Dorado, C. (2016). Efecto de la implementación de un programa de estimulación cognitiva en una población de adultos mayores institucionalizados en la ciudad de Bogotá. Revista Chilena de Neuropsicología, 11(1), 12-18.

Sistema Integral de Información de la Protección Social -SISPRO-. (2017). Indicadores 2017, Recuperado de: https://www.sispro.gov.co/

Zhang, K., Wang, J., Peng, G., Liu, P., He, F., Zhu, Z. \& Luo, B. (2019). Effect of cognitive training on episodic memory retrieval in amnestic mild cognitive impairment patients: study protocol for a clinical randomized controlled trial. Trials, 20(1), 26. 